\title{
LACUNARY FOURIER SERIES ON NONCOMMUTATIVE GROUPS
}

\author{
SIGURĐUR HELGASON
}

1. Introduction. In classical Fourier analysis lacunary series play a considerable role due to theorems of Kolmogoroff, Banach, Sidon and others. According to the usual definition a Fourier series $\sum a_{k} e^{i n_{k} x}$ is called lacunary if $n_{k+1} / n_{k}>\lambda(>1)$ for all $k$. This definition makes use of the ordering of the integers and does not immediately extend to two dimensions or to more general groups which have been recognized as a proper setting for large parts of Fourier Analysis.

Let $G$ be a compact group and as usual let $\hat{G}$ denote the set of equivalence classes of unitary irreducible representations of $G$. The set $\hat{G}$ has the following "hypergroup" structure: To each pair $\alpha, \beta \in \hat{G}$ there is attached a measure $\mu_{\alpha, \beta}$ on $\hat{G}$. This is determined by the decomposition of the Kronecker product $\alpha \otimes \beta$. In terms of this structure there is a natural duality between normal subgroups of $G$ and certain subhypergroups of $\hat{G}$. Some of the abelian Pontrjagin duality extends to this situation, although two nonisomorphic finite groups $G$ may have the same hypergroup structure of $\hat{G}$.

The purpose of this note is to point out how, in certain instances, the hypergroup structure of $\hat{G}$ is related to properties of Fourier expansions on $G$. In particular we give a definition of a lacunary Fourier series on $G$ in terms of $\hat{G}$. If $G$ is the circle group, our definition is formally quite different from the usual one but has similar implications. The definition is wide enough to cover the case of a series of the form $\sum a_{n} e^{i x_{n}}$, where $x_{n}$ are independent variables and a well known theorem of Kolmogoroff about such series can be extended to Fourier series on the product $\Pi_{n} U(n), U(n)$ denoting the unitary group in $n$ dimensions. Furthermore, the theorem of Banach stating that a lacunary $L^{1}$-series is an $L^{2}$-series is generalized to noncommutative groups.

2. The duality. We shall be concerned with compact groups $G$ with normalized Haar measure $d g$ and the familiar function spaces $L^{1}(G)$ and $L^{2}(G)$ of integrable and square integrable functions. The corresponding norms are denoted \|\|$_{1}$ and \|\|$_{2}$. Every function $f \in L^{1}(G)$ caln be uniquely represented by a Fourier series

Presented to the Society, October 27, 1956; received by the editors January 25, 1958. 


$$
f(g) \sim \sum_{x \in \hat{G}} d_{x} \operatorname{Tr}\left\langle A_{x} D_{x}(g)\right\rangle
$$

where $\operatorname{Tr}$ denotes the usual trace, $G$ is the set of equivalence classes of irreducible unitary representations of $G, D_{x}$ is a member of the class $\chi, d_{\chi}$ is the degree of $\chi$ and $A_{\chi}$ is the linear transformation determined by

$$
A_{x}=\int_{G} f(g) D_{x}\left(g^{-1}\right) d g .
$$

For the expansion above, the Schur-Peter-Weyl formula is valid

$$
\int_{G}|f(g)|^{2} d g=\sum_{\chi \in \hat{G}} d_{\chi} \operatorname{Tr}\left\langle A_{\chi} A_{\chi}^{*}\right\rangle
$$

finiteness of one side implying the finiteness of the other. ( $B^{*}$ denotes the adjoint of the operator $B$ ). In case $f$ is a central function, that is $f$ is invariant under inner automorphisms, the Fourier series (1) takes the form $f(g) \sim \sum a_{\chi} \chi(g)$ where $g \rightarrow \chi(g)$ is the character of the class $\chi$.

The series (1) we call absolutely convergent if $\sum d_{x}^{2}\left\|A_{x}\right\|<\infty,\|\|$ denoting the usual norm.

Definition 2.1. A set $S$ is called a (discrete) hypergroup if there is given a mapping $(\alpha, \beta) \rightarrow \mu_{\alpha, \beta}$ of $S \times S$ into the set of measures on $S$. A subset $T$ of the hypergroup $S$ is called a subhypergroup if all the measures $\mu_{\alpha, \beta}(\alpha, \beta \in T)$ have support contained in $T$.

If $G$ is abelian, $\hat{G}$ is a group, and if $G$ is nonabelian every tensor product $\alpha \otimes \beta$ for $\alpha, \beta \in \hat{G}$ has a direct decomposition into irreducible unitary components. This induces a hypergroup structure in $\hat{G}$. If $A$ and $B$ are two representations of $G$ we call $A$ and $B$ disjoint if no irreducible component of $A$ is equivalent to an irreducible component of $B$.

The identity transformation of arbitrary dimension will be called $E$ and the irreducible unit representation of $G$ will be called $I$. If $M$ is an arbitrary subset of $G$ we let $M^{\perp}$ stand for the set of classes $\alpha \in \hat{G}$ such that $D_{\alpha}(g)=E$ for each $g \in M$. Similarly if $\mathfrak{S} \in \hat{G}$ we let $\mathfrak{S}^{\perp}$ denote the subset of $G$ determined by the equations $D_{\alpha}(g)=E$ for each $\alpha \in \mathfrak{E}$. For simplification we call a subhypergroup $\mathfrak{S}$ of $\hat{G}$ a normal subhypergroup if $I \in \mathfrak{F}$ and if $\alpha \in \mathfrak{W}$ implies $\bar{\alpha} \in \mathfrak{W}$ (the bar denotes complex conjugation).

We have then the following duality between normal subhypergroups of $\hat{G}$ and closed normal subgroups of $G$. This is closely related to a duality outlined by van Kampen [3]. 
THEOREM 1.

(i) If $M \subset G, M^{\perp}$ is a normal subhypergroup of $\hat{G}$ and $\left(M^{\perp}\right)^{\perp}$ is the smallest closed normal subgroup of $G$ containing $M$.

(ii) If $\mathfrak{S} \subset \hat{G}, \mathfrak{S}^{\perp}$ is a closed normal subgroup of $G$ and $\left(\mathfrak{W}^{\perp}\right)^{\perp}$ is the smallest normal subhypergroup of $\hat{G}$ containing $\mathfrak{S}$.

(iii) If $N$ is a closed normal subgroup of $G,(G / N)^{\wedge}=N^{\perp}$.

Proof. (i) Let $\alpha, \beta \in M^{\perp}$ and let $D_{\alpha}, D_{\beta}$ be corresponding representations. From the direct decomposition of $D_{\alpha} \otimes D_{\beta}$ we get for the characters the decomposition

$$
\alpha(g) \beta(g)=\chi_{1}(g)+\cdots+\chi_{n}(g)
$$

where the $\chi_{i}$ are characters of irreducible representations whose dimensions $d_{i}$ satisfy

$$
d_{\alpha} \cdot d_{\beta}=d_{1}+\cdots+d_{n} .
$$

Now if $g \in M$ we have $\alpha(g)=d_{\alpha}$ and $\beta(g)=d_{\beta}$ and since $\max _{g}|\chi(g)|$ $=d_{\chi}$ we conclude from (4) and (5) that $\chi_{i}(g)=d_{i}$ for all $i$. It follows, that $D_{x_{i}}(g)=E$ so $\chi_{i} \in M^{\perp}$ and $M^{\perp}$ is a subhypergroup which is normal. It is obvious that $\left(M I^{\perp}\right)^{\perp}$ is a closed normal subgroup containing $M$. If $N$ is some arbitrary closed normal subgroup containing $M$, then $\left(M^{\perp}\right)^{\perp} \subset N$ because if $n \in\left(M^{\perp}\right)^{\perp}-N$ we can (by going to the factor group $G / N)$ find a representation $D \in \hat{G}$ such that $D(g)=E$ for $g \in N$ but $D(n) \neq E$. Then $D \in N^{\perp}-M^{\perp}$ which contradicts $M \subset N$.

(ii) If $\mathfrak{S C} \hat{G}$ it is clear that $\mathfrak{S}^{\perp}$ is a closed normal subgroup and $\left(\mathfrak{Q}^{\perp}\right)^{\perp}$ is a normal subhypergroup. The matrix elements from $\mathfrak{S}$ and $I$ can be regarded as a family of continuous functions on $G / \mathfrak{S}^{\perp}$ which separates points. Let $\Omega$ be the set of linear combinations of matrix elements from the normal subhypergroup $\mathfrak{S}^{*}$ generated by $\mathfrak{S}$ and $I$. By the Peter-Weyl theorem $Q$ is uniformly dense in the space of continuous functions on $G / \mathfrak{Q}^{\perp}$. Now if there were a representation $D \in \mathfrak{Q}^{\perp \perp}-\mathfrak{Q}^{*}$, each matrix element $a(g)$ from $D$ could be uniformly approximated by elements of $R$ but on the other hand $a(g)$ is orthogonal to $R$ by the orthogonality relations. This shows that $\mathfrak{S}^{*}=\mathfrak{S}^{\perp \perp}$.

(iii) Proof obvious.

\section{Multipliers.}

Definition 3.1. A hyperfunction on $G$ is a mapping which assigns to each $\chi \in \hat{G}$ a linear transformation of a complex vector space of dimension $d_{x}$.

Definition 3.2. A hyperfunction $\Gamma$ on $\hat{G}$ is called a multiplier if for each Fourier series for a continuous function

$$
f(g) \sim \sum d \chi \operatorname{Tr}\left\langle A_{\chi} D_{\chi}(g)\right\rangle
$$


the series

$$
f_{\Gamma}(g) \sim \sum d_{\chi} \operatorname{Tr}\left\langle\Gamma_{\chi} A_{\chi} D_{\chi}(g)\right\rangle
$$

is also a Fourier series for a continuous function.

It is easy to see from the closed graph theorem that if $\Gamma$ is a multiplier there exists a bounded measure $\mu_{\Gamma}$ on $G$ such that $f_{\Gamma}=f * \mu_{\Gamma}$ (convolution product). Less trivial is the following extension of a theorem of Sidon:

THEOREM 2. Let $\Gamma$ be a multiplier such that for each Fourier series (6) (with continuous $f$ ) the corresponding series (7) is absolutely convergent. Then there exists a function $F \in L^{2}(G)$ such that

$$
f_{\Gamma}=f * F \quad \text { for all continuous } f \text {. }
$$

Proof. Let $\Gamma$ be a multiplier with the properties stated in the theorem. Then $B \Gamma$ is also of that type provided $B$ is a hyperfunction on $\hat{G}$ satisfying $\sup _{x}\left\|B_{x}\right\|<\infty$. To see that $B \Gamma$ really is a multiplier we remark that an absolutely convergent series (in the sense defined in this paper) is uniformly convergent on $G$; this last fact is easily verified by writing each Fourier matrix $A_{\chi}$ as $P_{\chi} V_{\boldsymbol{\chi}}$ where $P_{\boldsymbol{\chi}}$ is positive definite and $V_{\chi}$ is unitary. Using a previous remark we see that there exist bounded measures $\mu_{\Gamma}$ and $\mu_{B \Gamma}$ on $G$ such that

$$
\begin{aligned}
f_{B} \Gamma & =f * \mu_{B} \Gamma, \\
f_{\Gamma} & =f * \mu \Gamma .
\end{aligned}
$$

The hyperfunctions $B$ satisfying $\sup _{x}\left\|B_{x}\right\|<\infty$ (the bounded hyperfunctions) form a Banach space under the norm $\sup _{x}\left\|B_{x}\right\|$. The mapping $T: B \rightarrow \mu_{B \Gamma}$ is a linear mapping of the Banach space of bounded hyperfunctions into the Banach space of measures on $G$ and again from the closed graph theorem it follows easily that this mapping is continuous. Now an integrable function on $G$ can be identified with a hyperfunction on $\hat{G}$ via the Fourier series expansion. If the function $\phi \in L^{1}(G)$ corresponds to $B$ in this manner we see that $\mu_{B \Gamma}$ is absolutely continuous with respect to Haar measure and has a derivative, say $\phi^{\Gamma} \in L^{1}(G)$. Then the mapping $\tilde{T}: \phi \rightarrow \phi^{\Gamma}$ is a linear transformation of $L^{1}(G)$ into itself and since $T$ above is continuous it follows that $\tilde{T}$ is spectrally continuous in the sense of [2]. Furthermore $\phi^{\Gamma}=\mu_{\Gamma} * \phi$ so $\tilde{T}$ commutes with right translations on $G$.

This being established, Theorem 2 follows from Theorem $A$ in [2] which is an extension of a theorem of Littlewood and states that the spectrally continuous operators that commute with right translations are precisely the left convolutions with $L^{2}$-functions on $G$. 
Definition 3.3. A subset $S \subset \hat{G}$ is called distinguished if for every Fourier series for a continuous function

$$
f(g) \sim \sum_{x \in \widehat{F}} d_{x} \operatorname{Tr}\left\langle A_{\chi} D_{x}(g)\right\rangle
$$

the subseries

$$
\sum_{x \in S} d_{x} \operatorname{Tr}\left\langle A_{\chi} D_{x}(g)\right\rangle
$$

also represents a continuous function $f_{S}$.

For abelian groups $G$, the distinguished sets were investigated in [1]. For the noncommutative case a partial description is given by

THEOREM 3. The distinguished sets that preserve positivity in the sense that $f_{S} \geqq 0$ whenever $f \geqq 0$ are precisely the normal subhypergroups of $\hat{G}$.

Proof. The mapping $f \rightarrow f_{S}$ is continuous (uniform topology) by the closed graph theorem and commutes with left translations. Hence there exists a bounded measure $\mu_{S}$ on $G$ such that $f_{S}=f * \mu_{S}$ for all $f$. The mapping $f \rightarrow f_{S}$ also commutes with right translations, so that the Fourier-Stieltjes series for $\mu_{S}$ has the form

$$
\mu_{S}(g) \sim \sum_{\chi \in S} d_{\chi} \chi(g)
$$

Using the assumption of the theorem we see that $\mu_{S}$ is a positive measure so by a theorem of Wendel [7] the condition $\mu_{S} * \mu_{S}=\mu_{S}$ implies that there exists a compact subgroup $K$ of $G$ such that $\mu_{S}(A)$ $=\mu(A \cap K)$ for every Borel set $A$, where $\mu$ is the Haar measure on $K$. From the Fourier-Stieltjes series for $\mu_{S}$ we see that

$$
\int_{G} \bar{D}_{\chi}(g) d \mu_{S}(g)=\int_{K} \bar{D}_{\chi}(k) d \mu(k)= \begin{cases}E & \text { if } \chi \in S, \\ 0 & \text { if } \chi \in S\end{cases}
$$

which implies $\bar{D}_{\chi}(k)=E$ for all $k$ if and only if $\chi \in S$ or otherwise expressed: $K^{\perp}=S$.

On the other hand, if $S$ is a normal subhypergroup then the Haar measure $\mu$ on $S^{\perp}$ extended to a measure on $G$ by $\mu(E)=\mu\left(E \cap S^{\perp}\right)$ has the Fourier-Stieltjes series

$$
\mu(g) \sim \sum_{\chi \in S} d_{\chi} \chi(g)
$$

and for each continuous function $f$ on $G$ with Fourier series (8) the continuous function $f * \mu$ has Fourier series (9), proving that $S$ is distinguished. 
4. Lacunary series. In this section we discuss extensions of theorems of Kolmogoroff and Banach.

Let $I$ be a set and to each element $i$ of $I$ attached an integer $d_{i}$. We consider the compact group $G=\prod_{i \in I} U\left(d_{i}\right)$ where $U(m)$ denotes the unitary group in $m$ dimensions. The projection $D_{i}$ of $G$ onto $U\left(d_{i}\right)$ is a unitary representation which clearly is irreducible, in other words $I$ can be regarded as a subset of $\widehat{G}$. We consider Fourier series of the form

$$
\sum_{i \in I} d_{i} \operatorname{Tr}\left\langle A_{i} D_{i}(g)\right\rangle
$$

and we shall now indicate the proof of the following theorem.

Theorem 4. Suppose $f \in L^{1}(G)$ and has a Fourier series of the form (10). Then $f \in L^{2}(G)$ and moreover $2^{-1 / 2}\|f\|_{2} \leqq\|f\|_{1} \leqq\|f\|_{2}$.

In the case where $d_{i}=1$ for each $i \in I$, this result is a well known theorem of Kolmogoroff.

The essence of Theorem 4 is proved in [2]. In fact let us consider a finite subset $J$ of $I$ and a series of the form

$$
s(g)=\sum_{j \in J} d_{j} \operatorname{Tr}\left\langle B_{j} D_{j}(g)\right\rangle .
$$

It is then clear that

$$
\int_{G}|s(g)|^{m} d g=\int_{V J}\left|\sum_{j} d_{j} \operatorname{Tr}\left\langle B_{j} D_{j}\right\rangle\right|^{m} d D_{J}
$$

where $d D_{J}$ denotes the Haar measure on the product $V_{J}=\prod_{j \in J} U\left(d_{j}\right)$. By the proof of Lemma 4.1 in [2] and the relation (4.12) in [2] we get

$$
\int_{G}|s(g)|^{4} d g \leqq 2\left[\int_{G}|s(g)|^{2} d g\right]^{2} .
$$

Using the inequality

$$
\left[\int|h(g)| d g\right]^{2} \geqq\left[\int|h(g)|^{4} d g\right]^{-1} \cdot\left[\int|h(g)|^{2} d g\right]^{3}
$$

which is a special case of Hölder's inequality we obtain

$$
\int_{G}|s(g)| d g \geqq 2^{-1 / 2}\left[\int_{G}|s(g)|^{2} d g\right]^{1 / 2} .
$$

By standard approximation arguments the function $f$ can be ap- 
proximated in $L^{1}$-norm by functions of the form (11), and (13) becomes valid for the function $f$. Theorem 4 is proved.

We shall now see that the set $I$ in the above situation appears as a lacunary subset of $\widehat{G}$ in a certain sense.

Let again $G$ be an arbitrary compact group. If $\alpha, \beta \in \hat{G}$ we denote by $D_{\alpha}$ and $D_{\beta}$ arbitrary members of $\alpha$ and $\beta$ respectively, $d_{\alpha}$ and $d_{\beta}$ the corresponding dimensions and $n_{\alpha, \beta}$ the number of irreducible components in $D_{\alpha} \otimes D_{\beta}$ (counted with multiplicity).

Definition 4.1. A subset $S \subset \hat{G}$ is called lacunary if the two following conditions are satisfied.

(I) Whenever $(\alpha, \beta)$ and $(\gamma, \delta)$ are different pairs from $S$ (that is, the characters $\alpha+\beta$ and $\gamma+\delta$ are different) $D_{\alpha} \otimes D_{\beta}$ and $D_{\gamma} \otimes D_{\delta}$ are disjoint.

(II) There exists a constant $K$ such that $n_{\alpha, \beta}<K$ for all $\alpha, \beta \in S$.

A Fourier series of the form $\sum_{x \in S} d_{\chi} \operatorname{Tr}\left\langle A_{\chi} D_{\chi}(g)\right\rangle$ is called lacunary if $S$ is lacunary.

The following statements show that the series (10) is indeed lacunary.

(i) $D_{i} \otimes D_{j}$ is irreducible if $i \neq j$.

(ii) If $d_{i}=1$ then $D_{i} \otimes D_{i}$ is irreducible.

(iii) If $d_{i} \geqq 2$ then $D_{i} \otimes D_{i}$ decomposes into two irreducible parts (of dimensions $\left(d_{i}^{2}+d_{i}\right) / 2$ and $\left(d_{i}^{2}-d_{i}\right) / 2$ ).

(iv) $D_{i} \otimes D_{i}$ is disjoint from $D_{j} \otimes D_{j}$ if $i \neq j$.

(i) and (ii) are obvious. (iii) is a corollary of Lemma 4.1 in [2] combined with the fact that the space of symmetric and antisymmetric tensors are left invariant by $D_{i} \otimes D_{i}$. Concerning (iv) we remark that the number of irreducible components common to $D_{i} \otimes D_{i}$ and $D_{j} \otimes D_{j}$ is equal to

$$
\int_{G}\left(\chi_{i}^{2} \bar{\chi}_{j}^{2}\right) d g=\int_{U\left(d_{i}\right) \times U\left(d_{i j}\right)}\left(\operatorname{Tr} D_{i}\right)^{2}\left(\operatorname{Tr} D_{j}^{-1}\right)^{2} d D_{i} d D_{j}
$$

( $d D_{m}$ is the Haar measure on $U\left(d_{m}\right)$ ), and this last integral vanishes as shown in the proof of the cited lemma.

For a general compact group we have a simple result in similar direction.

THEOREM 5. Let $f$ be a central function in $L^{1}(G)$ and suppose $f$ has a lacunary Fourier series. Then $f \in L^{2}(G)$.

PROOF. The Fourier expansion of $f$ can be written $f(g) \sim \sum_{\chi \in S} a_{\chi} \chi(g)$ where $a_{\chi}$ is a complex number and $S$ is lacunary. We consider a finite partial sum $s(g)=\sum_{1}^{N} a_{n} \chi_{n}(g)$. Then 


$$
s^{2}=\sum a_{p}^{2} \chi_{p}^{2}+2 \sum_{p>q} a_{p} a_{q} \chi_{p} \chi_{q} .
$$

If we here expand $\chi_{p}^{2}$ and $\chi_{p} \chi_{g}$ into a sum of characters the same $\chi_{i}$ will not occur more than once due to condition (I), and we get easily

$$
\begin{aligned}
\int_{Q}|s(g)|^{4} d g & =\sum_{1}^{N}\left|a_{p}\right|{ }^{4} n_{p, p}+2 \sum_{p>q}\left|a_{p}\right|^{2}\left|a_{q}\right|^{2} n_{p, q} \\
& \leqq K\left(\sum_{1}^{N}\left|a_{p}\right|^{2}\right)^{2} .
\end{aligned}
$$

Using the inequality (12) we obtain the conclusion $f \in L^{2}(G)$ in exactly the same manner as before.

Concerning the relation with the classical definition of lacunary series we remark that a series of the form $\sum a_{k} e^{i n_{k} x}$ where $n_{k+1} / n_{k}>2$ for all $k$, is lacunary in the sense of Definition 4.1. This is easily verified and is indeed a basic property in the proof of most classical theorems on lacunary series.

As a simple consequence of Theorem 5 we mention the following fact:

TheOREM 6. Let $G$ be an abelian group which is compact and not totally disconnected. Suppose the infinite series

$$
\sum a_{\chi} \sigma \cdot \chi(g)
$$

is a Fourier series for some $L^{1}$-function for each permutation $\sigma$ of $\hat{G}$. Then $\sum_{x \in \widehat{G}}\left|a_{x}\right|^{2}<\infty$.

Proof. By a theorem of Pontrjagin $\hat{G}$ has an infinite cyclic subgroup and therefore an infinite countable lacunary subset. Now we can write

$$
\sum_{a \chi \neq 0} a_{\chi} \chi(g)=\sum_{S} a_{\chi} \chi(g)+\sum_{T} a_{\chi} \chi(g)
$$

where both sets $S$ and $T$ are infinite and $\sum_{s}\left|a_{x}\right|<\infty$. Hence $\sum_{T} a_{\chi} \sigma \cdot \chi(g)$ is an $L^{1}$-series for every permutation $\sigma$ of $\hat{G}$. Choose this permutation in such a way that $\sum_{T} a_{\chi} \sigma \cdot \chi(g)$ is a lacunary series and apply Theorem 5. Q.E.D.

If $G$ is an arbitrary compact group, $\hat{G}$ need not possess any infinite lacunary subsets. As a simple example we mention $G=S U(2)$. The hypergroup structure of $\hat{G}$ is here described by the Clebsch-Gordan formula [8] which shows that the requirement (II) in Definition 4.1 is not fulfilled for any infinite subset $S \subset \bar{G}$. 


\section{BIBLIOGRAPHY}

0. S. Bochner, Über die Struktur von Fourierreihen fastperiodischer Funktionen, S. B. Math.-Nat. Kl. Bayer. Akad. Wiss. (1928) pp. 181-190.

1. S. Helgason, Some problems in theory of almost periodic functions, Math. Scand. vol. 3 (1955) pp. 49-67.

2. - Topologies of group algebras and a theorem of Littlewood, Trans. Amer. Math. Soc. vol. 86 (1957) pp. 269-283.

3. E. R. van Kampen, Almost periodic functions and compact groups, Ann. of Math. vol. 37 (1936) pp. 78-91.

4. L. Pontrjagin, Topological groups, Princeton, 1939.

5. S. Sidon, Ein Satz ïber die Fourierschen Reihen stetiger Funktionen, Math. Z. vol. 34 (1932) pp. 485-486.

6. S. Tannaka, Über den Dualitätssatz der nichtkommutativen topologischen Gruppen, Tôhoku Math. J. vol. 45 (1938) pp. 1-12.

7. J. G. Wendel, Haar measure and the semigroup of measures on a compact group, Proc. Amer. Math. Soc. vol. 5 (1954) pp. 923-929.

8. H. Weyl, Gruppentheorie und Quantenmechanik, Leipzig, 1928.

9. A. Zygmund, Trigonometrical series, Warszawa-Lwow, 1935.

University of Chicago 